
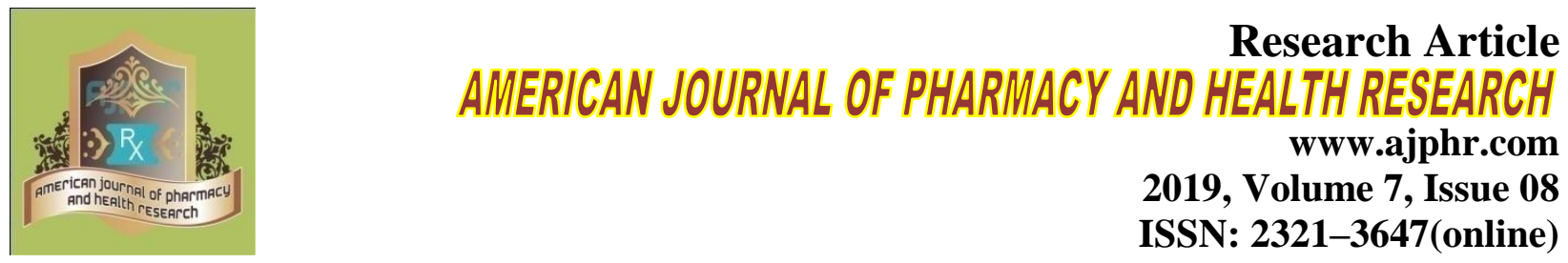

\title{
Design, Synthesis and Antimicrobial Activity of 2-Aromatic Substituted-1,3-Thaizolidine Derivatives
}

\author{
Krishna Reddy Gayam ${ }^{1 *}$, Senthilkumar Palaniappan ${ }^{2}$ \\ 1. Research Scholar, Department of Pharmaceutical Sciences, Sri Venkateshwara University, \\ Gajraula, Amroha, Uttar Pradesh-244236, India. \\ 2. Department of Pharmaceutical Chemistry, J.K.K.Nattraja College of Pharmacy, \\ Natarajapuram, NH-544 (Salem to Coimbatore), Kumarapalayam-638 183, Namakkal (Dt),
} Tamilnadu, India..

\begin{abstract}
A series of $N$-\{[2-(substituted methylidene)hydrazinyl](methylsulfanyl)methyl $\}$-2-substituted1,3-thiazolidine-4-carboxamide derivative were synthesized. The synthesized compounds were characterized by IR, NMR, Mass spectral data. The synthesized compounds were screened for their antifungal and antibacterial activity against pathogenic fungus and bacteria. Compounds TS-2, TS-4 and TS-9 exhibited good antibacterial and antifungal activity against the tested microorganisms, and the rest of the molecules shows good to moderate activity.
\end{abstract}

Keywords: substitute thiazolidine, microwave assisted synthesis, minimum inhibitory concentration, antibacterial, antifungal.

*Corresponding Author Email: krishnagm219@gmail.com

Received 11 July 2019, Accepted 17 July 2019

Please cite this article as: Gayam KR et al., Design, Synthesis and Antimicrobial Activity of 2Aromatic Substituted-1,3-Thaizolidine Derivatives. American Journal of Pharmacy \& Health Research 2019. 


\section{INTRODUCTION}

Thiazolidine derivatives containing aromatic ring plays an important role in modern medicinal chemistry, showing a wide range of biological activities such as antimicrobial, analgesic, antitubercular, antidepressant, anticancer, anti-inflammatory, anticonvulsant, hypolipidemic, analgesic, immunotropic activities and NPY receptor ligands binding. The prevalence of aromatic substituted thiazolidine cores in biologically active molecules has stimulated the need for elegant and efficient ways to make these heterocyclic lead thiazolidine as an important class of compounds for new drug development attracting much attention. The inevitable consequence of the widespread use of antimicrobial agents has been the emergence of antibiotic-resistant pathogens, fuelling an ever increasing need for new drugs. Several aromatic substituted thiazolidine derivatives have been synthesized as target structures and evaluated for their biological activities. The cytotoxicity of the reported compounds in the review indicate good safety associated with many of the thiazolidine derivatives ${ }^{[1-5]}$.

\section{Literature Review}

Pandeya et al $^{[6]}$ derived a series of Schiff base and Mannich bases, prepared from isatin. The synthesized compounds were evaluated for Antimicrobial activity by agar diffusion method.

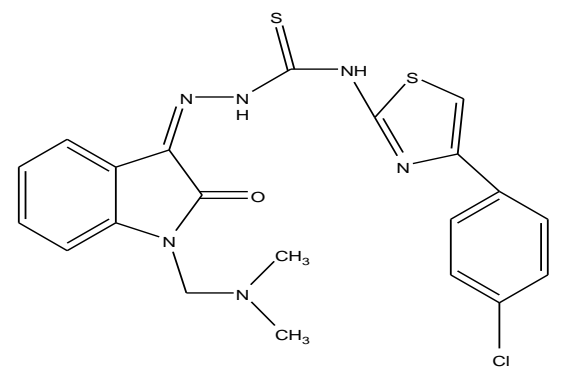

Ranjana et al ${ }^{[7]}$ prepared a series of phthalimido [ 2-aryl-3-(5'-(4"-pyridyl)-1',3',4'-thiadiazol-2'yl)-4-oxothiazolidin-5-yl] ethanoates. The synthesized compounds were analyzed for antimicrobial activity against Escherichia coli, Proteus vulgaris, Klebsiella pneumoniae, Pseudomonas auregenosa, Salmonella typhi and Bacillus subtilis bacterial strains by cup or well method.

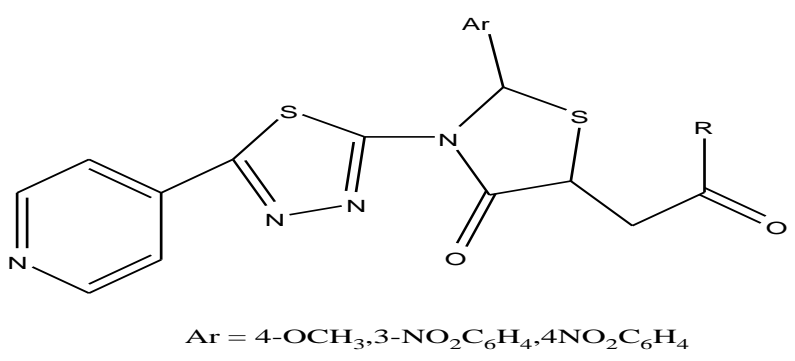


Meltem Ceylan et al ${ }^{[8]}$ prepared 3-(substituted-benzyl)-5-(4-chloro-2-piperidin-1yl-thiazole-5ylmethylene)-thiazolidine-2,4-dione derivatives and evaluated their antimicrobial activity against Staphylococcus aureus ATCC 250 and Escherichia coli.

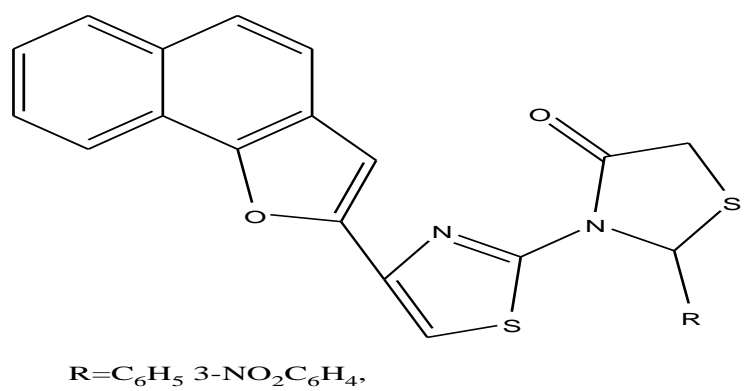

Bhoot D. P. et al ${ }^{[4]}$ prepared a series of 2-arylimino-3-aryl-5-[5'-(3,4-dichlorophenyl)2'furylidene]-4-thiazolidinones and analyzed their antimicrobial activity against $E$. coli, $P$. vulgaris.

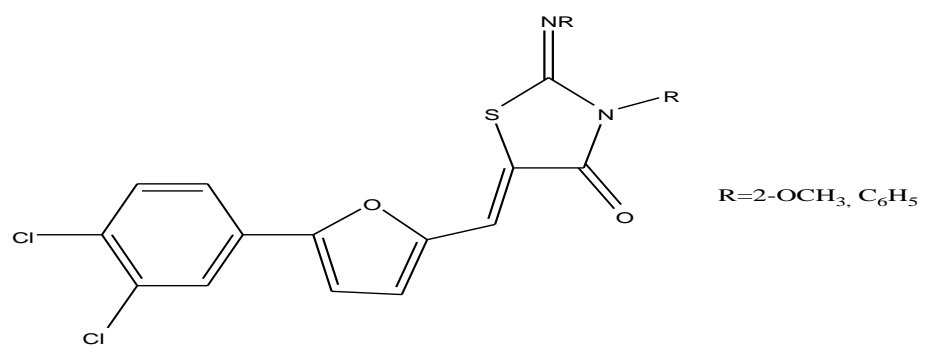

Sharma M. C. et al ${ }^{[9]}$ prepared a series of N-(5-methyl-4-oxo-thiazolidin-3-yl)-nicotinamide and analyzed the antimicrobial activity against B. subtilis, S. aureus, E. coli, A. niger and C. albicans.

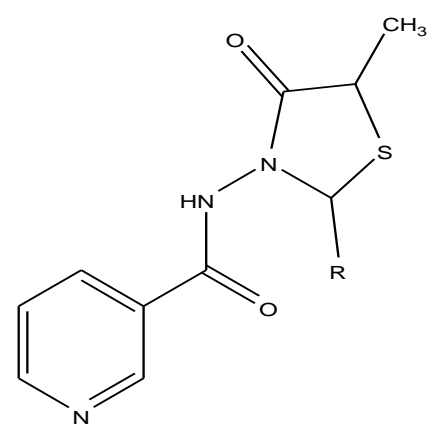

\section{Aim and Objective}

The purpose of the present work was to explore and develop novel molecules with improved potential for treating microbial infections. In this paper we report the design, synthesis and evaluation of antimicrobial activity of 2-aromatic substituted-1,3-thiazolidine-4-carboxamide derivatives. 


\section{MATERIALS AND METHOD}

\section{Synthesis of methyl thiourea from thiourea $(\mathrm{T} 1)^{[10]}$}

Thiourea (10g, 131mmol, 1meq) was dissolved in $100 \mathrm{ml}$ of methanol. Methyl iodide (8.21ml, $131 \mathrm{mmol}, 1 \mathrm{meq})$ was added to the reaction mixture and refluxed for $1 \mathrm{~h}$. After completion of the reaction, solvent was evaporated to yield crude product. This crude product was washed thrice with diethyl ether to yield the desired product as white solid.

\section{Synthesis of N-tert-butoxycarbonyl methyl thiourea (T2) ${ }^{[10]}$}

A solution of $\mathrm{BOC}_{2} \mathrm{O}(18.65 \mathrm{~g}, 124 \mathrm{mmol}, 1 \mathrm{meq})$ in $50 \mathrm{ml} \mathrm{DCM}$ was slowly added to a solution of methyl thiourea $(\mathrm{T} 1,28.0 \mathrm{~g}, 128.0 \mathrm{mmol}, 1 \mathrm{meq})$ and Triethylamine $(17.4 \mathrm{ml}, 124 \mathrm{mmol}, 1 \mathrm{meq})$ in $200 \mathrm{ml} \mathrm{DCM}$. After stirring for $20 \mathrm{~h}$ at room temperature, the reaction mixture was washed with water and brine and the organic phase was dried over magnesium sulphate. The solvent was evaporated under reduced pressure and crude product was formed.

\section{Synthesis of 2-Substituted-thiazolidine-4-carboxylic acid (T3) ${ }^{[12]}$}

A mixture of L-cysteine $(3.16 \mathrm{~g}, 26.11 \mathrm{mmol})$ and appropriate aldehydes $(26.15 \mathrm{mmol})$ in ethanol $(300 \mathrm{ml})$ and water $(30 \mathrm{ml})$ was stirred at room temperature for $6-15 \mathrm{~h}$, and the solid precipitated out was collected, washed with diethyl ether and dried to afford according (2RS, 4R)-2-arylthiazolidine-4-carboxylic acid .

Synthesis of N-(amino(methylthio)methyl)-2-substituted thiazolidine-4-carboxamide (T4) ${ }^{[11]}$ A mixture of 2-Substituted-thiazolidine-4-carboxylic acid (T3, 0.3-0.5g), DCC (1.2meq) and HOBT $(1.05 \mathrm{meq})$ in DCM $(200 \mathrm{ml})$ was stirred at room temperature for $10 \mathrm{~min}$. To this solution, tert-butyl amino(methylthio)methyl carbamate (1.05meq) and Triethyl amine (1.2 meq) were added and stirring continued at room temperature for $12-16 \mathrm{~h}$. The reaction mixture was diluted with DCM $(50 \mathrm{ml})$ and sequentially washed with water, satd. Sodium bicarbonate, brine and dried over magnesium sulphate. The solvent was removed under reduced pressure to yield tert-butyl (2-substituted thiazolidine-4-carboxamido) (methylthio)methylcarbamate as crude product, which were stirred with TFA (0.6-1.01) in $20 \mathrm{ml}$ DCM at room temperature for $1-8 \mathrm{~h}$ to cleave the BOC group. The reaction mixture was concentrated, washed with satd. Sodium bicarbonate and the solvent was removed to yield crude solid.

Synthesis of 2-substituted-[hydrazinyl(methylsulfanyl)methyl]-1,3-thiazolidine-4carboxamide (T5) ${ }^{[13]}$

Conventional Method: 
Concentrated $\mathrm{HCl}$ was added dropwise with stirring to hydrazine hydrate which was previously maintained at a temperature of $5-25^{\circ} \mathrm{C}$ followed by ethylene glycol $(25 \mathrm{ml})$. To the above reaction mixture $\mathrm{N}$-(amino(methylthio)methyl)-2-substituted thiazolidine-4-carboxamide (T4, $0.01 \mathrm{~mol}$ ) was added in small portions. The mixture was then refluxed for $2 \mathrm{~h}$ and then poured into crushed ice. The separated solid was then filtered and recrystallized from ethanol.

\section{Microwave Method:}

Concentrated $\mathrm{HCl}$ was added dropwise with stirring to hydrazine hydrate which was previously maintained at a temperature of $5-25^{\circ} \mathrm{C}$ followed by ethylene glycol $(22 \mathrm{ml})$. To the above reaction mixture $\mathrm{N}$-(amino(methylthio)methyl)-2-substituted thiazolidine-4-carboxamide (T4, $0.01 \mathrm{~mol}$ ) was added in small portions. The mixture was charged under microwave for 6 minutes and allowed to cool, poured into crushed ice. The separated solid was then filtered and recrystallized from ethanol.

\section{Synthesis of $N$-\{[2-(substituted methylidene)hydrazinyl](methylsulfanyl)methyl $\}$-2- substituted-1,3-thiazolidine-4-carboxamide (TS i-xxiv) ${ }^{[14]}$ \\ Conventional Method:}

To a solution of substituted aldehydes $(0.01 \mathrm{~mol})$ in methanol/ethanol $(15 \mathrm{ml})$, compound (T5, $0.01 \mathrm{~mol}$ ) was added along with few drops of acetic acid and the mixture was refluxed for $3 \mathrm{~h}$. The reaction mixture was cooled, then poured into ice-cold water and the separated solid was filtered, dried and recrystallized from ethanol.

\section{Microwave Method:}

To a solution of substituted aldehydes $(0.01 \mathrm{~mol})$ in methanol/ethanol $(15 \mathrm{ml})$, compound (T5, $0.01 \mathrm{~mol}$ ) and a few drops of acetic acid were added and the mixture was charged under microwave for 6-7 minutes. The reaction mixture was cooled, poured into ice-cold water and the separated solid was filtered, dried and recrystallized from ethanol.

\section{RESULTS AND DISCUSSION:}

In the present study, various 2-aromatic substituted-1,3-thiazolidine derivatives were synthesized. The structure of the synthesized compounds were confirmed by IR, NMR, Mass spectral data. The IR spectrum of all the synthesized compounds show bands in the region of 3150-3302 $\mathrm{cm}^{-1}$ and $1450-1750 \mathrm{~cm}^{-1}$ corresponding to $\mathrm{NH}$ and $\mathrm{C}=\mathrm{O}$. The IR spectrum in the region of 2950 to $3100 \mathrm{~cm}^{-1}$ shows the presence of $\mathrm{C}-\mathrm{H}$ in aliphatic and aromatic ring respectively. All the synthesized compounds show bands in the region of 1100 to $1400 \mathrm{~cm}^{-1}$ showing the presence of $\mathrm{C}-\mathrm{CH}_{3}, \mathrm{Ar} \mathrm{C}=\mathrm{C}$ and mono substituted phenyl ring. The NMR spectrum 
of all the compounds shows characteristic peak of for $\mathrm{CH}_{2}$ at $2.8 \delta \mathrm{ppm}$, the $\mathrm{NH}_{2}$ protons at $3.8 \delta$ ppm, for aromatic protons multiplet appears at 1.3 to $7.6 \delta \mathrm{ppm}$. The Mass spectra of the synthesized compounds were found to be corresponding with its $\mathrm{m} / \mathrm{z}$ mass. The synthesized compounds were screened for their in-vitro antimicrobial activity against various bacteria species like Salmonella paratyphi, Pseudomonas aeruginosa, Escherchia coli, Bacillus subtilis, Micrococcus luteus, Staphylococcus aureus, Klebsiella pneumonia, Staphylococcus albus and various fungal organisms like Aspergillus niger, Aspergillus fumigatus, Candida albicans and Monascus purpureus. Among the synthesized 2-substituted thiazolidine derivatives, compound TS-2, TS-4 and TS-9 shows good activity against both bacterial and fungal organisms with MIC value of $3.12 \mu \mathrm{g} / \mathrm{ml}$.

TS-i: M.P. $\left({ }^{\circ} \mathrm{C}\right)$ : 178-180; IR spectral values $\left(\mathrm{cm}^{-1}\right)$ : $3418.21\left(\mathrm{NH}_{2}\right.$ stretching), $2919.22\left(\mathrm{CH}_{3}\right.$ stretching Ali), 1686.93 ( $\mathrm{C}=\mathrm{N}$ stretching), 1489.7 ( $\mathrm{C}=\mathrm{C}$ stretching), 1462.7 ( $\mathrm{C}=\mathrm{O}$ stretching), 1380.78 (C-H bending in Ali), 1204.33 (C-N stretching), 1086.12 (N-N stretching), 756.38 (C-Cl in Ar), 824.3 (C-S stretching).

TS-ii: M.P. $\left({ }^{\circ} \mathrm{C}\right)$ : 182-184; IR spectral values $\left(\mathrm{cm}^{-1}\right)$ : $3419.17\left(\mathrm{NH}_{2}\right.$ stretching $), 2922.11\left(\mathrm{CH}_{3}\right.$ stretching Ali), 1489.7 (C=N stretching), 1382.23 (C-N stretching), 750.3 (C-Cl in Ar), 759.3 (monosub ring), 825.8 (C-S stretching); 1H NMR: 2.5 (s, H, NH), 6.9 (s, 1H, Ar group), 7.8-7.9 (m, 3H, ArH), 7.9 (m, 3H, thiazolidine); Mass (m/z): M-1=418.

TS-iii: M.P. $\left({ }^{\circ} \mathrm{C}\right)$ : 177-179; IR spectral values $\left(\mathrm{cm}^{-1}\right)$ : $3419.17\left(\mathrm{OH}\right.$ stretching), $2919.22\left(\mathrm{CH}_{3}\right.$ stretching Ali), 1685.96 (C=N stretching), 1489.7 ( $\mathrm{C}=\mathrm{O}$ stretching), 1382.71 (C-N stretching), 1204.33 (N-N stretching), 693.0 (C-Cl in Ar), 733.69 (disub ring), 870.36 (C-S stretching).

TS-iv: M.P. $\left({ }^{\circ} \mathrm{C}\right)$ : 184-186; IR spectral values $\left(\mathrm{cm}^{-1}\right): 3328.53\left(\mathrm{NH}_{2}\right.$ stretching $), 2923.56\left(\mathrm{CH}_{3}\right.$ stretching Ali), 1685.96 (C=N stretching), 1627.63 ( $\mathrm{C}=\mathrm{C}$ stretching), 1576.52 ( $\mathrm{C}=\mathrm{O}$ stretching), 1380.3 (C-H bending in Ali), 1313.2 (C-N stretching), 1204.3 (N-N stretching), 668.69 (C-Cl in Ar), 894.7 (C-S stretching).

TS-v: M.P. $\left({ }^{\circ} \mathrm{C}\right)$ : 188-190; IR spectral values $\left(\mathrm{cm}^{-1}\right)$ : $3446.17\left(\mathrm{NH}_{2}\right.$ stretching), $2925.0\left(\mathrm{CH}_{3}\right.$ stretching Ali), 1684.5 ( $\mathrm{C}=\mathrm{N}$ stretching), 1627.14 ( $\mathrm{C}=\mathrm{C}$ stretching), 1576.04 ( $\mathrm{C}=\mathrm{O}$ stretching), 668.69 ( $\mathrm{C}-\mathrm{Cl}$ in $\mathrm{Ar})$.

TS-vi: M.P. $\left({ }^{\circ} \mathrm{C}\right): 168-170 ;$ IR spectral values $\left(\mathrm{cm}^{-1}\right)$ : $3412.9\left(\mathrm{NH}_{2}\right.$ stretching), $3067.23(\mathrm{CH}$ stretching in Ar), $2927.0\left(\mathrm{CH}_{3}\right.$ stretching Ali), $1718.75(\mathrm{C}=\mathrm{N}$ stretching $), 1627.14(\mathrm{C}=\mathrm{C}$ stretching), 1563.02 ( $\mathrm{C}=\mathrm{O}$ stretching), 1379.3 (C-H bending in Ali), 1233.74 (C-N stretching), 1092.96 (N-N stretching), 643.14 (C-Cl in Ar). 
TS-vii: M.P. $\left({ }^{\circ} \mathrm{C}\right): 192-194$; IR spectral values $\left(\mathrm{cm}^{-1}\right): 3328.59\left(\mathrm{NH}_{2}\right.$ stretching $), 2927.41\left(\mathrm{CH}_{3}\right.$ stretching Ali), 1627.6 ( $\mathrm{C}=\mathrm{N}$ stretching), 1574.5 ( $\mathrm{C}=\mathrm{C}$ stretching), 1486.2 ( $\mathrm{C}=\mathrm{O}$ stretching), 1312.1 (C-N stretching), 1088.62 (N-N stretching), 641.25 (C-Cl in Ar), 886.71 (C-S stretching).

TS-viii: M.P. $\left({ }^{\circ} \mathrm{C}\right)$ : 182-184; IR spectral values $\left(\mathrm{cm}^{-1}\right)$ : $3328.53\left(\mathrm{NH}_{2}\right.$ stretching), $3041.19(\mathrm{CH}$ stretching in Ar), $2927.41\left(\mathrm{CH}_{3}\right.$ stretching Ali), $1575.08(\mathrm{C}=\mathrm{N}$ stretching $), 1538.4(\mathrm{C}=\mathrm{C}$ stretching), 1574 (C=O stretching), 1439.71 (C-N stretching), 892.39 (C-S stretching).

TS-ix: M.P. $\left({ }^{\circ} \mathrm{C}\right)$ : 192-194; IR spectral values $\left(\mathrm{cm}^{-1}\right)$ : $3327.67\left(\mathrm{NH}_{2}\right.$ stretching), $2924.5\left(\mathrm{CH}_{3}\right.$ stretching Ali), 1718.26 (C=N stretching), 1627.6 (C=C stretching), 1239.04 (C-N stretching), 775.24 (C-F in Ar).

TS-x: M.P. $\left({ }^{\circ} \mathrm{C}\right)$ : 184-186; IR spectral values $\left(\mathrm{cm}^{-1}\right)$ : $3379.6(\mathrm{OH}$ stretching), $3025.76(\mathrm{CH}$ stretching in Ar), $2927.86\left(\mathrm{CH}_{3}\right.$ stretching Ali), $1576.04(\mathrm{C}=\mathrm{N}$ stretching $), 1622.04(\mathrm{C}=\mathrm{C}$ stretching), 1088.14 (N-N stretching), 641.25 (C-Cl in Ar), 842.74 (C-S stretching); 1H NMR $(\delta$ values): 12.8 (bs, 1H, NH); 8.5 (bs, 1H, CONH); 7.9 (s, 1H, CH=N); 7.0 - $7.6(\mathrm{~m}, 10 \mathrm{H}, \mathrm{Ar}-\mathrm{H})$; 3.3 (s, 2H, CH2); 1.8 (s, 2H, Thiazolidine- $\mathrm{CH}_{2}$ ); Mass (m/z): M-2=450.

TS-xi: M.P. $\left({ }^{\circ} \mathrm{C}\right)$ : 192-194; IR spectral values $\left(\mathrm{cm}^{-1}\right)$ : 3114.47 (Ar C-H stretching), 1608.34 ( $\mathrm{C}=\mathrm{N}$ in $\mathrm{Ar}$ ), 1525.42 ( $\mathrm{C}=\mathrm{C}$ stretching), 1348.96 ( $\mathrm{NO}_{2}$ stretching), 1103.08 ( $\mathrm{S}-\mathrm{H}$ bending), 820.16 (di subs benzene), 718.35 (C-Cl stretching).

TS-xii: M.P. $\left({ }^{\circ} \mathrm{C}\right)$ : 188-190; IR spectral values $\left(\mathrm{cm}^{-1}\right): 3181.9\left(\mathrm{NH}_{2}\right.$ stretching $), 2921.63\left(\mathrm{CH}_{3}\right.$ stretching Ali), 1599.6 (C=O stretching), 1396.14 (C-H bending in Ali), 1290.14 (C-N stretching), 1027.8 (N-N stretching), 744.3 (C-Cl in Ar), 854.3 (C-S stretching).

TS-xiii: M.P. $\left({ }^{\circ} \mathrm{C}\right):$ 202-204; IR spectral values $\left(\mathrm{cm}^{-1}\right)$ : $3327.57\left(\mathrm{OH}\right.$ stretching), $3114.47\left(\mathrm{NH}_{2}\right.$ stretching), 3041.1 ( $\mathrm{CH}$ stretching in Ar), $2927.89\left(\mathrm{CH}_{3}\right.$ stretching Ali), $1628.11(\mathrm{C}=\mathrm{N}$ stretching), 1575.6 ( $\mathrm{C}=\mathrm{C}$ stretching), 1522.62 ( $\mathrm{C}=\mathrm{O}$ stretching), 1386.7 (C-N stretching), 1046.13 (N-N stretching), 848.04 (disub ring), 892.3 (C-S stretching).

TS-xiv: M.P. $\left({ }^{\circ} \mathrm{C}\right): 198-200$; IR spectral values $\left(\mathrm{cm}^{-1}\right)$ : $3414.55\left(\mathrm{OH}\right.$ stretching), $3337.5\left(\mathrm{NH}_{2}\right.$ stretching), 3056.6 ( $\mathrm{CH}$ stretching in Ar), $2925.4\left(\mathrm{CH}_{3}\right.$ stretching Ali), 1718.2 (C=N stretching), $1627.8(\mathrm{C}=\mathrm{C}$ stretching), 1582.31 ( $\mathrm{C}=\mathrm{O}$ stretching), 1379.82 (C-H bending in Ali), 1244.83 (C$\mathrm{N}$ stretching), 1086.12 (N-N stretching), 1453.1 (N=O stretching), 759.6 (disub ring), 889.9 (C-S stretching).

TS-xv: M.P. $\left({ }^{\circ} \mathrm{C}\right)$ : 184-186; IR spectral values $\left(\mathrm{cm}^{-1}\right)$ : $3435.56\left(\mathrm{OH}\right.$ stretching), $3114.47\left(\mathrm{NH}_{2}\right.$ stretching), 1532.12 (C=N stretching), 1482.2 (C=C stretching), 1340.2 (C-N stretching), 1039.4 (N-N stretching), 773.31 (C-Cl in Ar), 846.59 (disub ring), 911.2 (C-S stretching). 
TS-xvi: M.P. $\left({ }^{\circ} \mathrm{C}\right)$ : 188-190; IR spectral values $\left(\mathrm{cm}^{-1}\right)$ : 3181.9(NH2 stretching),2921.63(CH3 stretching Ali),1599.6(C=O stretching),1396.14(C-H bending in Ali),1290.14(C-N stretching),1027.8(N-N stretching),744.3(C-Cl in Ar),854.3( C-S stretching).

TS-xvii: M.P. $\left({ }^{\circ} \mathrm{C}\right)$ : 192-194; IR spectral values $\left(\mathrm{cm}^{-1}\right)$ : $3326.12\left(\mathrm{NH}_{2}\right.$ stretching), $3025.7(\mathrm{CH}$ stretching in Ar), $2926.9\left(\mathrm{CH}_{3}\right.$ stretching Ali), $1744.3(\mathrm{C}=\mathrm{N}$ stretching $), 1628.1 \quad(\mathrm{C}=\mathrm{C}$ stretching), 1576.04 ( $\mathrm{C}=\mathrm{O}$ stretching), 1312.8 (C-N stretching), 1092.9 (C-O-C stretching), 641.2 (disub ing), 894.8 (C-S stretching).

TS-xviii: M.P. $\left({ }^{\circ} \mathrm{C}\right)$ : 184-186; IR spectral values $\left(\mathrm{cm}^{-1}\right): 3416.46$ (OH stretching), $3213.79\left(\mathrm{NH}_{2}\right.$ stretching), 3035.4 ( $\mathrm{CH}$ stretching in $\mathrm{Ar}$ ), 1650 ( $\mathrm{C}=\mathrm{N}$ stretching), 1517.7 ( $\mathrm{C}=\mathrm{C}$ stretching), 1340.28 (C=S stretching), 1399.1 (C-N stretching), 1108.8 (C-O-C stretching), 852.38 (C-S stretching).

TS-xix: M.P. $\left({ }^{\circ} \mathrm{C}\right)$ : 168-170; IR spectral values $\left(\mathrm{cm}^{-1}\right)$ : $3327.57\left(\mathrm{NH}_{2}\right.$ stretching), $2927.41\left(\mathrm{CH}_{3}\right.$ stretching Ali), 1723.09 ( $\mathrm{C}=\mathrm{N}$ stretching), 1628.5 ( $\mathrm{C}=\mathrm{C}$ stretching), 1572.8 ( $\mathrm{C}=\mathrm{O}$ stretching), 1327.7 (C=S stretching), 1259.2 (C-N stretching), 1040.8 (C-O-C stretching), 756.64 (disub ing), 889.0 (C-S stretching).

TS-xx: M.P. $\left({ }^{\circ} \mathrm{C}\right)$ : 192-194; IR spectral values $\left(\mathrm{cm}^{-1}\right): 3420.14\left(\mathrm{NH}_{2}\right.$ stretching), $3100.0(\mathrm{CH}$ stretching in Ar), 1629.55 ( $\mathrm{C}=\mathrm{N}$ stretching), 1541.8 ( $\mathrm{C}=\mathrm{O}$ stretching), 1422.8 ( $\mathrm{C}=\mathrm{S}$ stretching), 1346.05 (C-N stretching), 1107.0 (C-O-C stretching), 722.21 (C-Cl in Ar), 851.4 (disub ring), 927 (C-S stretching)

TS-xxi: M.P. $\left({ }^{\circ} \mathrm{C}\right)$ : 196-198; IR spectral values $\left(\mathrm{cm}^{-1}\right)$ : 3413.3 (OH stretching), $3327.5\left(\mathrm{NH}_{2}\right.$ stretching), $3067.23(\mathrm{CH}$ stretching in $\mathrm{Ar}), 2927.41\left(\mathrm{CH}_{3}\right.$ stretching Ali), $1718.2(\mathrm{C}=\mathrm{N}$ stretching), 1626.8 ( $\mathrm{C}=\mathrm{C}$ stretching), 1573.6 (C=O stretching), 1371.03 (C-N stretching), 1191.7 (C-O-C stretching), 775.3 (disub ring).

TS-xxii: M.P. $\left({ }^{\circ} \mathrm{C}\right)$ : 188-190; IR spectral values $\left(\mathrm{cm}^{-1}\right)$ : $3244.6\left(\mathrm{NH}_{2}\right.$ stretching $), 2926.4\left(\mathrm{CH}_{3}\right.$ stretching Ali), 1712.4 ( $\mathrm{C}=\mathrm{N}$ stretching), 1624.7 ( $\mathrm{C}=\mathrm{C}$ stretching), 1563.02 ( $\mathrm{C}=\mathrm{O}$ stretching), 1343.86 (C-N stretching), 1113.69 (C-O-C stretching), 769.45 (disub ring)

TS-xxiii: M.P. $\left({ }^{\circ} \mathrm{C}\right)$ : 184-186; IR spectral values $\left(\mathrm{cm}^{-1}\right): 3413.3\left(\mathrm{NH}_{2}\right.$ stretching), $3062.4(\mathrm{CH}$ stretching in Ar), $2926.45\left(\mathrm{CH}_{3}\right.$ stretching Ali), $1718.2 \quad(\mathrm{C}=\mathrm{N}$ stretching $), 1627.1 \quad(\mathrm{C}=\mathrm{C}$ stretching), 1562.05 ( $\mathrm{C}=\mathrm{O}$ stretching), 1374.03 (C-H bending in Ali), 1238.5 (C-N stretching), 1046.1 (C-O-C stretching), 889.5 (C-S stretching).

TS-xxiv: M.P. $\left({ }^{\circ} \mathrm{C}\right): 192-194$; IR spectral values $\left(\mathrm{cm}^{-1}\right): 3416.28$ (OH stretching), $3208.7\left(\mathrm{NH}_{2}\right.$ stretching), 3073.01 ( $\mathrm{CH}$ stretching in $\mathrm{Ar}), 2958.27\left(\mathrm{CH}_{3}\right.$ stretching Ali), $1728.87(\mathrm{C}=\mathrm{N}$ 
stretching), 1640.16 ( $\mathrm{C}=\mathrm{C}$ stretching), 1561.09 ( $\mathrm{C}=\mathrm{O}$ stretching), 1388.0 (C=S stretching), 1251.54 (C-N stretching), 1088.27 (C-O-C stretching), 884.20 (C-S stretching).

\section{Scheme:}

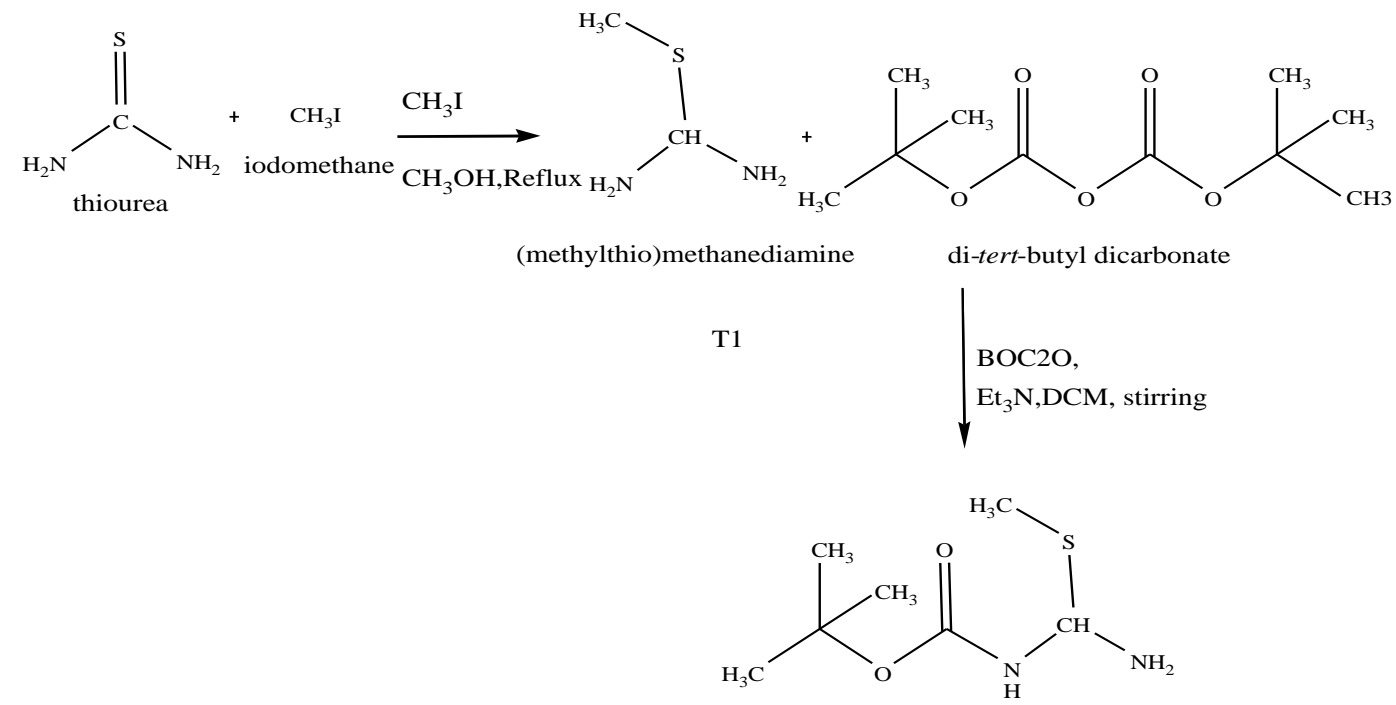

tert-butyl amino(methylthio)methylcarbamate

$\underset{+}{\mathrm{T} 2}$

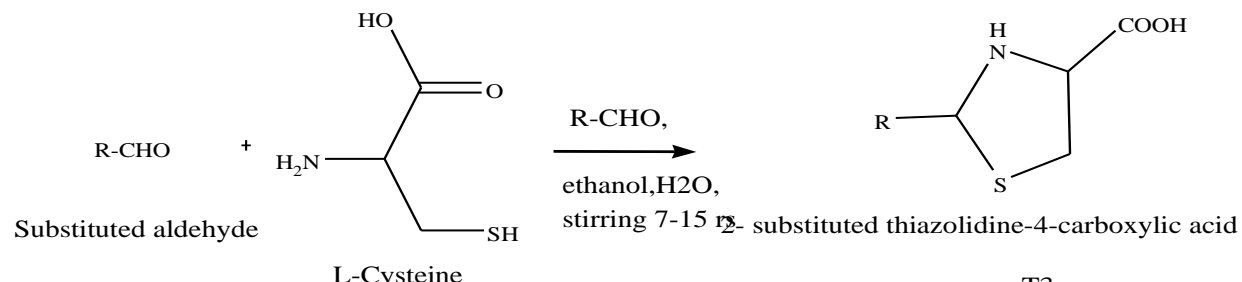

L-Cysteine

T3

EDC.HCl, HOBT, Et3N, DCM stirring 8- 12hrs.<smiles>CSC(N)NC(=O)C1CSCN1</smiles>

$N$-(amino(methylthio)methyl)-2-substituted thiazolidine-4-carboxamide
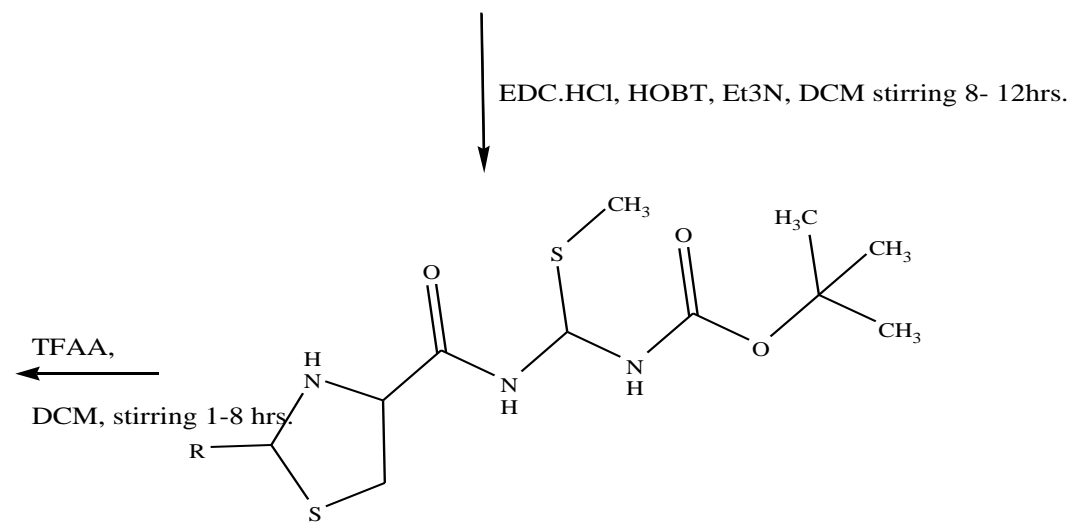

tert-butyl (2-substituted thiazolidine-4-carboxamido)(methylthio)methylcarbamate 


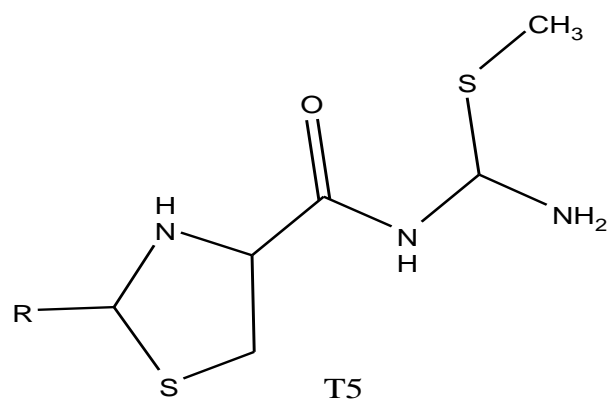

$N$-(amino(methylthio)methyl)-2-substituted thiazolidine-4-carboxamide

$\mathrm{NH}_{2} \mathrm{NH}_{2} . \mathrm{HCl}$,

ethylene glycol,

MVI-5-8 min,

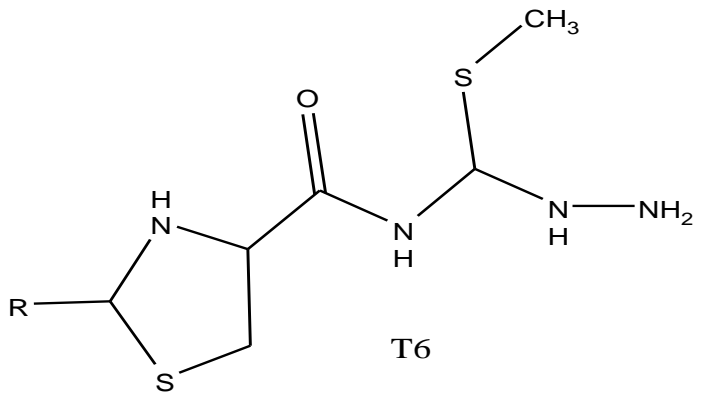

2-substituted-[hydrazinyl(methylsulfanyl)methyl]-1,3-thiazolidine-4-carboxamide

$\mathrm{R}_{1}$-CHO, ethanol,

glacial. $\mathrm{CH}_{3} \mathrm{COOH}$,

MVI-5-8 min, 180W.

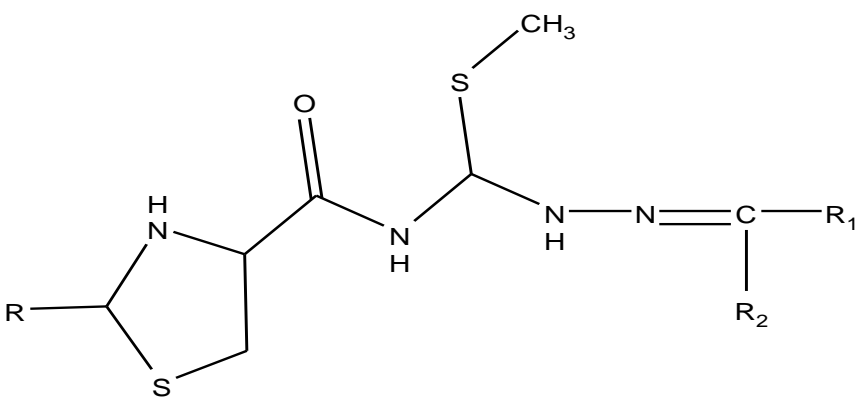

TS i-xxiv

$N$-\{[2-(substituted methylidene)hydrazinyl](methylsulfanyl)methyl $\}$-2-substituted -1,3-thiazolidine-4-carboxamide 
Table 1: General structure and physical data of synthesized compounds.

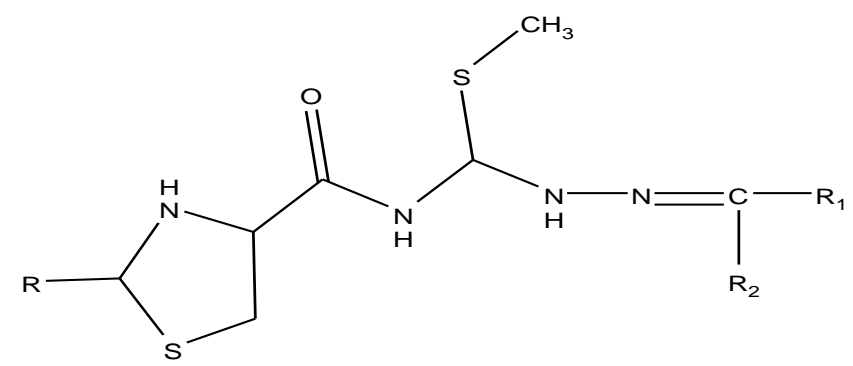

\begin{tabular}{|c|c|c|c|c|c|c|}
\hline Compd & $\mathbf{R}$ & $\mathbf{R}_{1}$ & $\mathbf{R}_{2}$ & Mol. Formula & $\begin{array}{l}\text { Mol. } \\
\text { Weight }\end{array}$ & cLog $P$ \\
\hline TS-1 & $4-\mathrm{Cl}-\mathrm{C}_{6} \mathrm{H}_{4-}$ & $4-\mathrm{Cl}-\mathrm{C}_{6} \mathrm{H}_{4-}$ & $-\mathrm{H}$ & $\mathrm{C}_{19} \mathrm{H}_{20} \mathrm{~N}_{4} \mathrm{OS}_{2} \mathrm{Cl}_{2}$ & 454.02 & 3.879 \\
\hline TS-2 & $4-\mathrm{Cl}-\mathrm{C}_{6} \mathrm{H}_{4-}$ & $\mathrm{C}_{6} \mathrm{H}_{5-}$ & $-\mathrm{H}$ & $\mathrm{C}_{19} \mathrm{H}_{21} \mathrm{~N}_{4} \mathrm{OS}_{2} \mathrm{Cl}$ & 420.08 & 3.05 \\
\hline TS-3 & $4-\mathrm{Cl}-\mathrm{C}_{6} \mathrm{H}_{4-}$ & $2-\mathrm{OH}-\mathrm{C}_{6} \mathrm{H}_{4-}$ & $-\mathrm{H}$ & $\mathrm{C}_{19} \mathrm{H}_{21} \mathrm{~N}_{4} \mathrm{O}_{2} \mathrm{~S}_{2} \mathrm{Cl}$ & 436.08 & 1.47 \\
\hline TS-4 & $4-\mathrm{Cl}-\mathrm{C}_{6} \mathrm{H}_{4-}^{-}$ & $4-\mathrm{OCH}_{3}-\mathrm{C}_{6} \mathrm{H}_{4-}^{-}$ & $-\mathrm{H}$ & $\mathrm{C}_{20} \mathrm{H}_{23} \mathrm{~N}_{4} \mathrm{O}_{2} \mathrm{~S}_{2} \mathrm{Cl}$ & 450.10 & 3.49 \\
\hline TS-5 & $4-\mathrm{Cl}-\mathrm{C}_{6} \mathrm{H}_{4-}$ & 3,4-di- $-\mathrm{OCH}_{3}-\mathrm{C}_{6} \mathrm{H}_{3}-$ & $-\mathrm{H}$ & $\mathrm{C}_{21} \mathrm{H}_{25} \mathrm{~N}_{4} \mathrm{O}_{3} \mathrm{~S}_{2} \mathrm{Cl}$ & 480.11 & 3.17 \\
\hline TS-6 & $4-\mathrm{Cl}-\mathrm{C}_{6} \mathrm{H}_{4-}^{-}$ & $4-\mathrm{CH}_{3}-\mathrm{C}_{6} \mathrm{H}_{4-}$ & $-\mathrm{H}$ & $\mathrm{C}_{20} \mathrm{H}_{23} \mathrm{~N}_{4} \mathrm{OS}_{2} \mathrm{Cl}$ & 434.10 & 3.55 \\
\hline TS-7 & $4-\mathrm{Cl}-\mathrm{C}_{6} \mathrm{H}_{4-}^{-}$ & $-\mathrm{H}$ & $-\mathrm{CH}_{3}$ & $\mathrm{C}_{20} \mathrm{H}_{23} \mathrm{~N}_{4} \mathrm{OS}_{2} \mathrm{Cl}$ & 434.10 & 3.17 \\
\hline TS-8 & $4-\mathrm{Cl}-\mathrm{C}_{6} \mathrm{H}_{4-}^{-}$ & $4-\mathrm{OCH}_{3}-\mathrm{C}_{6} \mathrm{H}_{4-}^{-}$ & $-\mathrm{CH}_{3}$ & $\mathrm{C}_{21} \mathrm{H}_{25} \mathrm{~N}_{4} \mathrm{O}_{2} \mathrm{~S}_{2} \mathrm{Cl}$ & 464.11 & 3.94 \\
\hline TS-9 & $4-\mathrm{Cl}-\mathrm{C}_{6} \mathrm{H}_{4-}^{-}$ & 4-F- $\mathrm{C}_{6} \mathrm{H}_{4-}$ & $-\mathrm{CH}_{3}$ & $\mathrm{C}_{20} \mathrm{H}_{22} \mathrm{~N}_{4} \mathrm{OS}_{2} \mathrm{ClF}$ & 452.09 & 4.06 \\
\hline TS-10 & $4-\mathrm{Cl}-\mathrm{C}_{6} \mathrm{H}_{4-}^{-}$ & $2-\mathrm{OH}-\mathrm{C}_{6} \mathrm{H}_{4-}$ & $-\mathrm{CH}_{3}$ & $\mathrm{C}_{20} \mathrm{H}_{23} \mathrm{~N}_{4} \mathrm{O}_{2} \mathrm{~S}_{2} \mathrm{Cl}$ & 450.10 & 2.34 \\
\hline TS-11 & $4-\mathrm{Cl}-\mathrm{C}_{6} \mathrm{H}_{4-}$ & $4-\mathrm{NO}_{2}-\mathrm{C}_{6} \mathrm{H}_{4-}^{-}$ & $-\mathrm{CH}_{3}$ & $\mathrm{C}_{20} \mathrm{H}_{22} \mathrm{~N}_{5} \mathrm{O}_{3} \mathrm{~S}_{2} \mathrm{Cl}$ & 479.09 & 3.66 \\
\hline TS-12 & $\mathrm{C}_{6} \mathrm{H}_{5^{-}}$ & $4-\mathrm{Cl}-\mathrm{C}_{6} \mathrm{H}_{4-}$ & $-\mathrm{H}$ & $\mathrm{C}_{19} \mathrm{H}_{21} \mathrm{~N}_{4} \mathrm{OS}_{2} \mathrm{Cl}$ & 420.98 & 3.16 \\
\hline TS-13 & $2-\mathrm{OH}-\mathrm{C}_{6} \mathrm{H}_{4}-$ & $2-\mathrm{OH}-\mathrm{C}_{6} \mathrm{H}_{4-}$ & $-\mathrm{CH}_{3}$ & $\mathrm{C}_{20} \mathrm{H}_{24} \mathrm{~N}_{4} \mathrm{O}_{3} \mathrm{~S}_{2}$ & 432.13 & 0.91 \\
\hline TS-14 & $2-\mathrm{OH}-\mathrm{C}_{6} \mathrm{H}_{4-}$ & $4-\mathrm{NO}_{2}-\mathrm{C}_{6} \mathrm{H}_{4-}$ & $-\mathrm{CH}_{3}$ & $\mathrm{C}_{20} \mathrm{H}_{23} \mathrm{~N}_{5} \mathrm{O}_{4} \mathrm{~S}_{2}$ & 461.12 & 2.23 \\
\hline TS-15 & $2-\mathrm{OH}-\mathrm{C}_{6} \mathrm{H}_{4-}^{-}$ & $4-\mathrm{Cl}-\mathrm{C}_{6} \mathrm{H}_{4}-$ & $-\mathrm{CH}_{3}$ & $\mathrm{C}_{20} \mathrm{H}_{23} \mathrm{~N}_{4} \mathrm{O}_{2} \mathrm{~S}_{2} \mathrm{Cl}$ & 450.10 & 3.20 \\
\hline TS-16 & $4-\mathrm{OCH}_{3}-\mathrm{C}_{6} \mathrm{H}_{4}-$ & $4-\mathrm{Cl}-\mathrm{C}_{6} \mathrm{H}_{4}-$ & $-\mathrm{H}$ & $\mathrm{C}_{20} \mathrm{H}_{23} \mathrm{~N}_{4} \mathrm{O}_{2} \mathrm{~S}_{2} \mathrm{Cl}$ & 451.0 & 3.7 \\
\hline TS-17 & $4-\mathrm{OCH}_{3}-\mathrm{C}_{6} \mathrm{H}_{4}-$ & $\mathrm{C}_{6} \mathrm{H}_{5-}^{-}$ & $-\mathrm{H}$ & $\mathrm{C}_{20} \mathrm{H}_{24} \mathrm{~N}_{4} \mathrm{O}_{2} \mathrm{~S}_{2}$ & 416.56 & 2.87 \\
\hline TS-18 & $\mathrm{C}_{6} \mathrm{H}_{5^{-}}$ & $4-\mathrm{OCH}_{3}-\mathrm{C}_{6} \mathrm{H}_{4-}^{-}$ & $-\mathrm{CH}_{3}$ & $\mathrm{C}_{21} \mathrm{H}_{26} \mathrm{~N}_{4} \mathrm{O}_{2} \mathrm{~S}_{2}$ & 430.15 & 3.22 \\
\hline TS-19 & $-\mathrm{C}_{6} \mathrm{H}_{5-}^{-}$ & $2-\mathrm{OH}-\mathrm{C}_{6} \mathrm{H}_{4}-$ & $-\mathrm{CH}_{3}$ & $\mathrm{C}_{20} \mathrm{H}_{24} \mathrm{~N}_{4} \mathrm{O}_{2} \mathrm{~S}_{2}$ & 416.13 & 1.63 \\
\hline TS-20 & $\mathrm{C}_{6} \mathrm{H}_{5-}^{-}$ & 4-F- $\mathrm{C}_{6} \mathrm{H}_{4-}$ & $-\mathrm{CH}_{3}$ & $\mathrm{C}_{20} \mathrm{H}_{23} \mathrm{~N}_{4} \mathrm{OFS}_{2}$ & 418.13 & 3.35 \\
\hline TS-21 & $4-\mathrm{OCH}_{3}-\mathrm{C}_{6} \mathrm{H}_{4-}$ & $2-\mathrm{OH}-\mathrm{C}_{6} \mathrm{H}_{4-}$ & $-\mathrm{H}$ & $\mathrm{C}_{20} \mathrm{H}_{24} \mathrm{~N}_{4} \mathrm{O}_{3} \mathrm{~S}_{2}$ & 432.55 & 1.29 \\
\hline TS-22 & $4-\mathrm{OCH}_{3}-\mathrm{C}_{6} \mathrm{H}_{4}-$ & $4-\mathrm{OCH}_{3}-\mathrm{C}_{6} \mathrm{H}_{4}-$ & $-\mathrm{H}$ & $\mathrm{C}_{21} \mathrm{H}_{26} \mathrm{~N}_{4} \mathrm{O}_{3} \mathrm{~S}_{2}$ & 446.58 & 2.84 \\
\hline TS-23 & $4-\mathrm{OCH}_{3}-\mathrm{C}_{6} \mathrm{H}_{4}-$ & 3,4-di- $\mathrm{OCH}_{3}-\mathrm{C}_{6} \mathrm{H}_{3}-$ & $-\mathrm{H}$ & $\mathrm{C}_{22} \mathrm{H}_{28} \mathrm{~N}_{4} \mathrm{O}_{4} \mathrm{~S}_{2}$ & 476.61 & 2.70 \\
\hline TS-24 & $\mathrm{C}_{6} \mathrm{H}_{5-}^{-}$ & $4-\mathrm{Cl}-\mathrm{C}_{6} \mathrm{H}_{4}-$ & $-\mathrm{CH}_{3}$ & $\mathrm{C}_{20} \mathrm{H}_{23} \mathrm{~N}_{4} \mathrm{OS}_{2} \mathrm{Cl}$ & 434.10 & 3.92 \\
\hline
\end{tabular}


Table 2: Minimum Inhibitory Concentration of Compounds

\begin{tabular}{|c|c|c|c|c|c|c|c|c|c|c|c|c|}
\hline \multirow[t]{2}{*}{$\begin{array}{l}\text { Micro } \\
\text { organism }\end{array}$} & \multicolumn{12}{|c|}{$\begin{array}{l}\text { Minimum Inhibitory Concentration }(\mu \mathrm{g} / \mathrm{mL}) \\
\text { Compound }\end{array}$} \\
\hline & TS-i & TS-ii & TS-iii & TS-iv & TS-v & TS-vi & TS-vii & TS-viii & TS-ix & TS-x & TS-xi & TS-xii \\
\hline S. albus & 6.25 & 3.17 & 12.5 & 6.25 & 12.5 & 6.25 & 12.5 & 6.25 & 6.25 & 12.5 & 12.5 & 6.25 \\
\hline B. subtilis & 6.25 & 6.25 & 12.5 & 6.25 & 12.5 & 6.25 & 12.5 & 6.25 & 6.25 & 12.5 & 12.5 & 6.25 \\
\hline S. aureus & 6.25 & 3.17 & 25 & 6.25 & 12.5 & 6.25 & 25 & 3.17 & 6.25 & 12.5 & 12.5 & 12.5 \\
\hline M. luteus & 6.25 & 6.25 & 25 & 12.5 & 12.5 & 6.25 & 25 & 6.25 & 6.25 & 12.5 & 12.5 & 12.5 \\
\hline E. coli & 12.5 & 3.17 & 25 & 6.25 & 25 & 12.5 & 25 & 3.17 & 3.17 & 25 & 12.5 & 12.5 \\
\hline S. paratyphi & 12.5 & 6.25 & 25 & 6.25 & 25 & 3.17 & 12.5 & 6.25 & 12.5 & 25 & 25 & 6.25 \\
\hline P. auroginosa & 6.25 & 3.17 & 6.25 & 6.25 & 6.25 & 12.5 & 12.5 & 6.25 & 6.25 & 25 & 6.25 & 6.25 \\
\hline K. pneumonia & 6.25 & 6.25 & 6.25 & 3.17 & 12.5 & 12.5 & 12.5 & 6.25 & 6.25 & 6.25 & 25 & 12.5 \\
\hline C. albicans & 12.5 & 3.17 & 6.25 & 3.17 & 12.5 & 3.17 & 12.5 & 3.17 & 6.25 & 12.5 & 25 & 12.5 \\
\hline A. niger & 12.5 & 6.25 & 6.25 & 3.17 & 12.5 & 12.5 & 12.5 & 6.25 & 3.17 & 12.5 & 25 & 12.5 \\
\hline A. fumigates & 12.5 & 12.5 & 12.5 & 6.25 & 25 & 12.5 & 12.5 & 6.25 & 12.5 & 12.5 & 25 & 12.5 \\
\hline M. purpureus & 12.5 & 6.25 & 12.5 & 3.17 & 25 & 3.17 & 12.5 & 6.25 & 12.5 & 12.5 & 25 & 12.5 \\
\hline
\end{tabular}

Solvent: DMSO; Media: Muller Hinton Broth,

Table 3: Minimum Inhibitory Concentration of Compounds

\begin{tabular}{|c|c|c|c|c|c|c|c|c|c|c|c|c|}
\hline \multirow[t]{3}{*}{ Micro-organism } & \multicolumn{12}{|c|}{ Minimum Inhibitory Concentration $(\mu \mathrm{g} / \mathrm{mL})$} \\
\hline & \multicolumn{12}{|c|}{ Compound } \\
\hline & TS xiii & TS-xix & TS-Xv & TS-xvi & TS-xvii & TS-xviii & TS-xix & TS-xx & TS-xxi & TS-xxii & TS-xxiii & TS-xxiv \\
\hline S. albus & 3.17 & 6.25 & 12.5 & 6.25 & 6.25 & 6.25 & 6.25 & 25 & 6.25 & 25 & 6.25 & 3.17 \\
\hline B. subtilis & 6.25 & 12.5 & 12.5 & 6.25 & 6.25 & 6.25 & 6.25 & 25 & 12.5 & 25 & 3.17 & 6.25 \\
\hline S. aureus & 6.25 & 12.5 & 25 & 6.25 & 6.25 & 6.25 & 3.17 & 50 & 12.5 & 12.5 & 6.25 & 6.25 \\
\hline M. luteus & 3.17 & 12.5 & 25 & 6.25 & 6.25 & 6.25 & 6.25 & 12.5 & 12.5 & 12.5 & 3.17 & 6.25 \\
\hline E. coli & 6.25 & 12.5 & 25 & 3.17 & 6.25 & 6.25 & 6.25 & 12.5 & 25 & 12.5 & 6.25 & 3.17 \\
\hline S. paratyphi & 3.17 & 6.25 & 12.5 & 6.25 & 6.25 & 6.25 & 12.5 & 25 & 12.5 & 25 & 3.17 & 6.25 \\
\hline P. auroginosa & 6.25 & 6.25 & 12.5 & 6.25 & 12.5 & 6.25 & 6.25 & 12.5 & 25 & 12.5 & 6.25 & 3.17 \\
\hline K. pneumonia & 6.25 & 6.25 & 25 & 6.25 & 6.25 & 6.25 & 3.17 & 12.5 & 50 & 25 & 3.17 & 6.25 \\
\hline C. albicans & 12.5 & 12.5 & 25 & 3.17 & 3.17 & 12.5 & 6.25 & 25 & 25 & 12.5 & 6.25 & 6.25 \\
\hline A. niger & 12.5 & 12.5 & 25 & 6.25 & 6.25 & 12.5 & 6.25 & 25 & 25 & 25 & 3.17 & 6.25 \\
\hline
\end{tabular}




\begin{tabular}{|c|c|c|c|c|c|c|c|c|c|c|c|c|}
\hline A. fumigates & 12.5 & 12.5 & 25 & 3.17 & 3.17 & 12.5 & 6.25 & 25 & 50 & 25 & 6.25 & 6.25 \\
\hline M. purpureus & 12.5 & 12.5 & 25 & 3.17 & 6.25 & 12.5 & 3.17 & 12.5 & 50 & 12.5 & 6.25 & 3.17 \\
\hline
\end{tabular}




\section{ACKNOWLEDGEMENT:}

The authors are thankful to Dept. of Science and Technology (DST) for funding the research.

\section{REFERENCES:}

1. Pandeya S.N, Sriram D., Nath G., DeClerq E. Synthesis, antibacterial, antifungal and anti-HIV activities of Schiff and Mannich bases derived from isatin derivatives and N-[4(4'-chlorophenyl)thiazol-2-yl] thiosemicarbazide. Eur. J. Pharm. Sci. 1999; 9: 25-31.

2. Amin K.M, Rahman A.D.E., Al-Eryani Y.A. Synthesis and preliminary evaluation of some substituted coumarins as anticonvulsant agents. Bioorg. Med. Chem. 2008; 16: 5377-5388.

3. Vagdevi H M, Vaidya V P, Latha K P, Padmashali B. Synthesis and pharmacological examination of some thiazolidinone derivatives of naphtha[2,1-b]furan. Indian J. Pharm. Sci 2006;68:719-725.

4. Bhoot D P, Khunt R C, Shankhavara V K. Journal of Sciences, Islamic Republic of Iran 2006;4: 17:323-325.

5. Paola V, Athina G, Matteo I, Franca Z. 2-Heteroarylimino-5-benzylidene-4thiazolidinones analogues of 2-thiazolylimino-5-benzylidene-4-thiazolidinones with antimicrobial activity: synthesis and structure-activity relationship. Bioorg. \& Med. Chem. 2008: 3714-3724.

6. Pandeya S.N, Sriram D., Nath G., DeClerq. Synthesis, antibacterial, antifungal and antiHIV activities of Schiff and Mannich bases derived from isatin derivatives and N-[4-(4'chlorophenyl)thiazol-2-yl] thiosemicarbazide. Eur. J. Pharm. Sci 1999; 9: 25-31.

7. Sharma R, Devendra P Nagda, Ganpat L Talesara, Synthesis of various isoniazidothiazolidinones and their imidoxy derivatives of potential biological interest Arkivoc (2006)1-12.

8. Ceylan M. Turk. Synthesis and Antimicrobial Activity of Some New 3-Substituted Benzyl-5-(4-chloro-2-piperidin-1ylthiazole-5-yl-methylene)-thiazolidine-2,4-dione

Derivatives. J. Chem, 30 (2006) 355 -360.

9. .Sharma M C, Sahua K, Kohalia Qsar, Synthesis And Biological Activity Studies Of Some Thiazolidinones Derivatives. Dig. J. Nanomaterials and Biostructures. (2009) 223 232. 
10. Kraus, A.; Ghorai, P.; Birnkammer, T.; Schnell, D.; Elz, S.; Seifert, R.; Dove, S.; Bernhardt, G.;Buschauer, A. NG-acylated aminothiazolyl propylguanidines as potent and selective histamine:H2 receptor agonists. ChemMedChem 2009; 4:232-240.

11. Park, J. H.; Choi, J. K.; Lee, E.; Lee, J. K.; Rhim, H.; Seo, S. H.; Kim, Y.; Doddareddy, M. R.; Pae, A. N.; Kang, J.; Roh, E. J. Lead discovery and optimization of T-type calcium channel blockers. Bioorg. Med. Chem. 2007; 15: 1409-1419.

12. Lu Y, Wang Z, Li C-M, Li W, Dalton JT, Miller DD. Synthesis and biological evaluation of 2-arylthiazolidine-4-caboxylic acid amides for melanoma and prostate cancer. Abstracts of Papers, 234 ${ }^{\text {th }}$ ACS National Meeting, Boston, MA, United States August 1923;2007:MEDI-304.

13. Reddy, L.V.; Suman, A.; Beevi, S.S.; Mangamoori, L.N.; Mukkanti, K.; Pal, S. Design and synthesis of 1-aroyl-2-ylidene hydrazines under conventional and microwave irradiation conditions and their cytotoxic activities. J. Braz. Chem. Soc. 2010;21: 98-104.

14. Boschelli, D.H.; Connor, D.T.; Flynn, D.L.; Sircar, J.C.; Hoefle, M.L. Triazole derivatives of fenamates as anti-inflammatory agents. US Patent 4962119, 1990. 